\section{Estimando el efecto del cambio climático sobre el riesgo de la enfermedad de Chagas en Chile por medio del número reproductivo}

\author{
SALVADOR AYALA ${ }^{1, \mathrm{a}}$, SERGIO ALVARADO ${ }^{2,6, \mathrm{~b}}$, DANTE CÁCERES $^{3,6, \mathrm{c}}$, \\ INÉS ZULANTAY ${ }^{4, d}$, MAURICIO CANALS ${ }^{5}$
}

\section{Effects of climate change on reproductive number of Chagas disease}

Background: Reproductive number $\left(R_{0}\right)$-maps estimate risk zones of vector-borne diseases and geographical distribution changes under climate change. Aim: To map $R_{0}$ aiming to estimate the epidemiological risk of Chagas disease in Chile, its distribution and possible changes due to the global climate change. Material and Methods: We used a relationship between $R_{0}$ and entomological parameters of vectors as function of environmental variables, to map the risk of Chagas disease in Chile, under current and projected future environmental conditions. Results: We obtained a geographical $R_{0}$ estimation of Chagas disease in Chile. The highest $R_{0}$ averages correspond to the Central-Northern regions of Chile. $T$. cruzi transmission area could increase in the future due to climate changes. Independent of the future condition, both for optimistic and pessimistic climate change scenarios, the area of potential risk for Chagas disease transmission would increase. The estimated $R_{0}$ values suggest that, if a control of $T$. infestans is not maintained, Chagas disease endemic status will persist or increase, independently of the climate change scenarios. Conclusions: Mapping $R_{0}$ values is an effective method to assess the risk of Chagas disease. The eventual increase in the transmission area of the disease is worrisome.

(Rev Med Chile 2019; 147: 683-692)

Key words: Basic Reproduction Number; Chagas disease; Risk.
'Sub-departamento de Vigilancia de Laboratorio, Instituto de Salud Pública de Chile (ISP). Santiago, Chile.

2Programa de Bioestadística, Escuela de Salud Pública, Facultad de Medicina, Universidad de Chile. Santiago, Chile. ${ }^{3}$ Programa de Salud Ambiental, Escuela de Salud Pública, Facultad de Medicina, Universidad de Chile. Santiago, Chile. ${ }^{4}$ Laboratorio de Parasitología, ICBM, Facultad de Medicina, Universidad de Chile. Santiago, Chile.

${ }^{5}$ Programa de Salud Ambiental, Escuela de Salud Pública, Departamento de

Medicina, Facultad de Medicina, Universidad de Chile. Santiago, Chile. ${ }^{6}$ Facultad de Ciencias de la Salud. Universidad de Tarapacá. Arica, Chile. ${ }^{a} G$ Gógrafo, Ms Salud Pública.

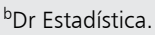

'M. Veterinario, Dr Salud Pública.

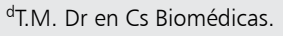

Trabajo financiado por Fondecyt 1150514 a MCL.

Loa autores declaran no tener conflictos de interés.

Recibido el 6 de agosto de 2018, aceptado el 24 de abril de 2019.

Correspondencia a:

Mauricio Canals

Programa de Salud Ambiental, Escuela

de Salud Pública \& Departamento

de Medicina, Facultad de Medicina, Universidad de Chile.

Independencia 939. Santiago, Chile.

Zip code 8380453.

www. mauriciocanals.cl.

mcanals@uchile.cl
I as enfermedades transmitidas por vectores son afectadas por el cambio climático alterando la distribución y tamaño de la población del reservorio y el vector ${ }^{1-8}$. Se ha propuesto que la enfermedad de Chagas no muestra una relación fuerte con las variaciones climáticas a corto plazo $^{9}$, pero las poblaciones de triatominos $\operatorname{si}^{10-15}$.

La presencia de un vector no es el único determinante del riesgo de enfermedad en la población. Existen otros factores como las densidades de po- blación, las tasas de picada, la esperanza de vida del vector y los hospederos, las poblaciones humana $\mathrm{y}$ animal y los factores sociales que son relevantes para determinar el riesgo de una enfermedad ${ }^{16}$, lo que se puede hacer calculando y mapeando el número reproductivo $\left(R_{0}\right)$ que representa el número de casos nuevos a partir de un caso en el tiempo infectante ${ }^{4,5,16-18}$.

La enfermedad de Chagas es considerada una de las principales enfermedades desatendidas, siendo la enfermedad emergente más importante 
de América ${ }^{19,20}$ afectando entre 6 y 11 millones de personas ${ }^{21,22}$. En Chile el vector biológico que transmite la enfermedad de Chagas se extiende entre Arica-Parinacota y Libertador Bernardo O’Higgins, con una población en riesgo eleva$\mathrm{da}^{22}$. La prevalencia de infección por T. cruzi es $0,7 \%, 1,5 \%$ en las zonas rurales y $0,6 \%$ en las zonas urbanas. En Chile, la transmisión vectorial de $T$. cruzi por T. infestans se ha interrumpido desde $1999^{22}$.

Se ha reportado la distribución de los vectore $^{23,24}$ y se ha correlacionado la distribución del vector principal $T$. infestans, estimado por ocurrencias, con la distribución de casos de enfermedad de Chagas y su variación potencial debido al cambio climático, proponiendo una probable reducción mínima de las zonas de riesgo ${ }^{8}$. Sin embargo, este último enfoque no considera los parámetros entomológicos y de poblaciones humanas que son variables en todo Chile.

El objetivo de este estudio es estimar el riesgo de la enfermedad de Chagas en Chile, su distribución y posibles cambios ante el cambio climático global, mediante la estimación y el mapeo de $R_{0}$, que es un estimador del riesgo de transmisión vectorial de T. cruzi.

\section{Material y Métodos}

El estudio abarcó entre Arica y Parinacota (18³0’S) y la Región de O’Higgins (34³ 36’S). La estimación de $R_{0}$ se basó en Canals et al. ${ }^{17}$ :

$$
R_{0 V}(T, P)=\frac{(1+v) b(T)^{2} f f^{\prime} d(T, P)}{(\mu+\alpha) \mu_{V}(T)}[1]
$$

Donde $v$ mide la población de reservorios animales $(v), b(T)$ es la tasa de picada en función de la temperatura $(T), f$ y $f$ 'representan la proporción de picadas en el hospedero infectado que hace un vector sano y del vector infectado a un hospedero susceptible; $d(T, P)$ es la densidad del vector (insectos/humano) en función de la temperatura y la precipitación $(P),(\mu+\alpha)$ es la suma de la tasa de mortalidad general y específica por Chagas y $\mu V(T)$ es la tasa de mortalidad del vector. El efecto del reservorio no se consideró $(v=0)$, y los parámetros $f, f^{\prime}, \mu$ y $\alpha$ se obtuvieron de la literatura: $f=0,01, f^{\prime}=0,001, \mu=0,0000457$ días $^{-1}$ y $\alpha=0,0000274$ días $^{-1}{ }^{16}$. Estos parámetros se pueden expresar en un factor $F=0,137$, obteniendo:

$$
R_{0 V}(T, P)=0,137 \frac{\mathrm{b}(T)^{2} \mathrm{~d}(T, P)}{\mu_{V}(T)}
$$

La tasa de mortalidad $\left(\mu_{V}(T)\right)$ se estimó siguiendo dos cohortes $(\mathrm{n}=20)$ de T. infestans en cámaras climáticas termoreguladas a $12{ }^{\circ} \mathrm{C}$ y 18 ${ }^{\circ} \mathrm{C}$ y una humedad relativa de $45 \%$ durante 12 meses, alimentadas naturalmente cada 2 semanas con sangre de gallinas, registrando semanalmente el número de individuos vivos. Además, se utilizó una cohorte seguida con la misma metodología a $25{ }^{\circ} \mathrm{C}^{11}$. Se ajustó una regresión: ln $(100 \mathrm{~N} /$ $\mathrm{N} 0)=b_{0}+b_{1} t$, ( $\mathrm{t}$ en meses), obteniendo la tasa de mortalidad que corresponde a la pendiente de decaimiento de los supervivientes $\left(\mu_{V}=\left|b_{1}\right|\right)$ en cada temperatura. Se ajustó una regresión cuadrática entre la tasa de mortalidad y la temperatura: $\left(\mu_{V}(T)=c_{0}+c_{1} T+c_{2} T^{2}\right)$.

La densidad de $T$. infestans $(d(T, P))$ se estimó en 50 localidades con una relación previamente obtenida ${ }^{11}$ :

$$
d=\frac{i_{t} i_{d} N_{v} K}{N}[3]
$$

donde $i$ es el índice triatomino (insectos capturados/individuo hora), $i_{d}$ el porcentaje de infestación domiciliaria, $N_{v}$ el número de viviendas, $N$ la población humana y $K$ un factor de corrección: número máximo de insectos capturados en una vivienda/índice tratomino máximo: $K=6.043 / 480=12,59^{11}$.

Los parámetros $i_{t}$ y $i_{d}$ se obtuvieron de estudios de infestación domiciliaria ${ }^{11,25-31} \cdot N_{v}$ y $N$ se obtuvieron del INE. Se obtuvo información para 50 comunas diferentes de la literatura (58 registros), y se ajustó una regresión cuadrática: $d(T, P)=a_{0}$ $+a_{1} T+a_{2} T^{2}+a_{3} P+a_{4} P^{2}$.

La tasa de picada $b(T)$ se estimó a través de la relación entre la tasa de picada y la tasa metabólica (gasto de energía, equivalente a la producción de $\left.\mathrm{CO}_{2}\right)$ en artrópodos $(32,33)$. Se obtuvieron 23 (13 hembras y 10 machos adultos) de T. infestans (masa corporal: $\mathrm{Mb}=175,5 \pm 75,6 \mathrm{mg}$; media \pm desviación estándar) de laboratorio. Se 
mantuvieron bajo un ciclo de Luz-Oscuridad de $12: 12 \mathrm{~h}$ a $24 \pm 2,5^{\circ} \mathrm{C}$ y $50 \pm 8 \%$ de humedad relativa, y se alimentaron sobre gallinas cada dos semanas. Después de 3 días sin comida, se midió la producción de $\mathrm{CO}_{2}\left(\mathrm{VCO}_{2}\right)$ a $10,5^{\circ} \mathrm{C}, 15^{\circ} \mathrm{C}, 23$ ${ }^{\circ} \mathrm{C}, 28^{\circ} \mathrm{C}$ y $32,5^{\circ} \mathrm{C}$ en una cámara metabólica ${ }^{34,35}$. Como la tasa metabólica está relacionada con la masa corporal: $V_{\mathrm{CO} 2}=M b e^{-E a / k T}$, donde $E a$ es la energía de activación enzimática y $k$ es la constante de Boltzman ${ }^{36}$, se ajustó una regresión entre $V_{\mathrm{CO} 2} \mathrm{y}$ $1 / T: \ln V_{\mathrm{CO}_{2}}=\ln (\mathrm{Mb})$-cte $(1 / \mathrm{T})$. Se convirtieron los valores de $V_{\mathrm{CO} 2}$ a valores de tasa de picada utilizando un factor de corrección igual a la relación entre la tasa de picada y el metabolismo a $20^{\circ} \mathrm{C}$ $(=0,0754 / 435,16=0,00017)$.

El valor de $R_{0}$ para cada precipitación y temperatura se obtuvo incorporando las relaciones obtenidas para $\mu_{V}(T), d(T, P)$ y $b(T)$ en [1].

Los valores ambientales se recopilaron de WorldClim (http://www.worldclim.org/) ${ }^{37}$ con resolución espacial de 2,5 grados (latitud/longitud). Los valores de $R_{0}$ se mapearon para tres escenarios utilizando SIG: 1) Situación actual: promedio1960-1990; 2) Escenario RCP 2,6: considerado un escenario optimista con baja emisión de gases de efecto invernadero (GEI), lo que conduce a elevaciones moderadas de elevación de la temperatura promedio; 3) Escenario RCP 8,5: escenario pesimista, altas emisiones de GEI.

Los resultados se analizaron mediante estadística descriptiva y se comparó la situación actual con los escenarios futuros utilizando la prueba de Kolmogórov-Smirnov.

\section{Resultados}

Hubo un buen ajuste entre la supervivencia y el tiempo en cada cohorte (Tabla 1 ) y la relación obtenida para la relación entre la mortalidad del vector $\left(\mu_{\mathrm{V}}(T)\right)$ y la temperatura $(T)$ fue: $\mu_{V}(T)=0,072-0,0076 T+0,00024 T^{2}\left(\mathrm{R}^{2} \approx 1\right)$ (Figura 1).

Los valores más altos de densidad de insectos se obtuvieron en Antofagasta, Coquimbo y RM (Tabla 2). Para la relación con temperatura y precipitación se obtuvo:

$d(T, P)=-328,5+44,9 T-1,42 T^{2}+$ $0,16 P-0,00026 P^{2} \quad\left(\mathrm{~F}_{4,52}=5,92, \mathrm{p}=0,0005\right.$; $\left.\mathrm{R}^{2}=0,31\right)$. La densidad de vectores varió entre 0,64 y 41,1, con media $22,45 \pm 12,86$ ( \pm desviación estándar). Los valores más altos $(d(T, P)>30)$ se obtuvieron con temperaturas de $14,3{ }^{\circ} \mathrm{C}\left(13,1^{\circ} \mathrm{C}\right.$ $16,3^{\circ} \mathrm{C}$ ) y una precipitación anual de $284,13 \mathrm{~mm}$ $(38,7 \mathrm{~mm}-443,6 \mathrm{~mm})$ (Figura 1).

Se obtuvo un aumento en la tasa metabólica en función de la temperatura, (Tabla 3). Para la relación con la temperatura se obtuvo $\ln V_{\mathrm{CO}^{2}}=17,6-3387,8(1 / \mathrm{T})\left(\mathrm{F}_{1,3}=19,8\right.$, $\left.\mathrm{p}=0,021 ; \mathrm{R}^{2}=0,82\right)$. El factor de corrección $(=0,0754 / 435,16=0,00017327)$ permitió estimar la relación entre la tasa de picada y la temperatura (Figura 1). El máximo para la tasa de picada $\mathrm{b}=0,226$ día $^{-1}$ se encontró a $32,5^{\circ} \mathrm{C}$, y el mínimo fue $b=0,096$ día $^{-1}$ a $10,5^{\circ} \mathrm{C}$.

Los valores de $R_{0}$ variaron de 0,021 a 9,6. Los valores más altos $\left(R_{0}>6\right)$ se encontraron entre los $14{ }^{\circ} \mathrm{C} \mathrm{y} 19{ }^{\circ} \mathrm{C}$, y entre $100 \mathrm{~mm}$ y $500 \mathrm{~mm}$ de Pp (Figura 1). Los mayores valores de mortalidad se ubicaron hacia los sectores cordilleranos del territorio. La densidad promedio de T. infestans fue de 13,8 individuos/humano. La mayor densidad se concentró en la zona central del país: Atacama, Coquimbo, Valparaíso y R. Metropolitana. Las mayores tasa de picada se ubicaron entre Arica y Parinacota y Atacama.

El valor de $R_{0}$ en el área de estudio fue de 2,33 $\pm 2,57$, una mediana de 1,01 . Los valores más altos de $R_{0}$ se localizaron en la zona central del país, entre Coquimbo y la RM (Figura 2).

\section{Tabla 1. Tasa de mortalidad $\left(\mu_{v}=\left|b_{1}\right|\right)$ en función de la temperatura de aclimatación (T) para tres cohortes de Triatoma infestans $\left(\ln \left(100 N / N_{0}\right)=b_{0}+b_{1} t\right.$, donde $t$ es el tiempo de desarrollo de cada cohorte)}

\begin{tabular}{|cccccc|}
\hline $\mathbf{T}\left({ }^{\circ} \mathbf{C}\right)$ & $\mathbf{n}$ & $\boldsymbol{\mu V}$ & Error St. & p-valor & IC 95\% \\
\hline 12 & 20 & 0,015 & 0,0026 & $<0,001$ & $0,009-0,021$ \\
18 & 20 & 0,012 & 0,0021 & $<0,001$ & $0,007-0,017$ \\
25 & 20 & 0,030 & 0,0021 & $<0,001$ & $0,025-0,035$ \\
\hline
\end{tabular}




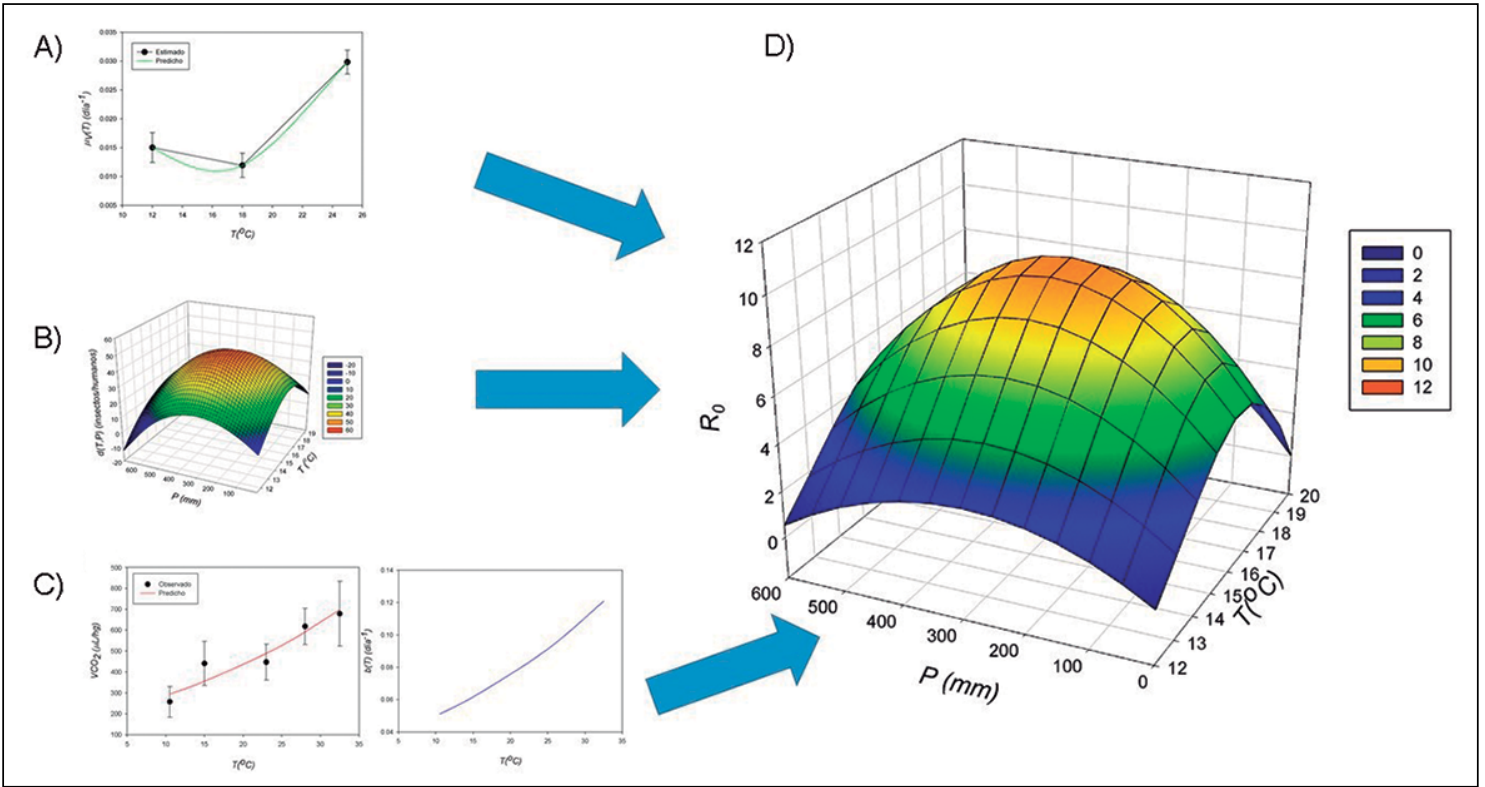

Figura 1. A) Variación de la tasa de mortalidad $\left(\mu_{v}(T)\right)$ con la temperatura $(T)$. Los puntos negros y las barras indican el promedio y la desviación estándar. En verde la curva ajustada. B) Curva ajustada mostrando la relación entre la densidad de vectores $(d(T, P))$ y la temperatura ambiental $(T)$ y precipitación $(P)$. C) Variación de la producción de $\mathrm{CO}_{2}\left(V_{\mathrm{CO} 2}(\mu \mathrm{L} / \mathrm{hg})\right)$ y la temperatura y curva ajustada entre la tasa de picada estimada $\left(b(T)\right.$ día $\left.^{-1}\right)$ y temperatura. D) Curva ajustada mostrando la relación entre el número reproductivo $\left(R_{0}\right)$ y la temperatura ambiental y precipitación.

Tabla 2. Valores promedio de parámetros entomológicos y densidad estimada de individuos de Triatoma infestans, para la zona endémica de la enfermedad de Chagas en Chile

\begin{tabular}{|lcccccccc|}
\hline \multicolumn{1}{c}{ Región } & $\boldsymbol{n}$ & $\boldsymbol{i}_{\boldsymbol{t}}$ & $\boldsymbol{i}_{\boldsymbol{d}}$ & $\boldsymbol{N}_{\mathbf{v}}$ & $\boldsymbol{N}$ & $\boldsymbol{d}$ & $\boldsymbol{T}\left({ }^{\circ} \mathbf{C}\right)$ & $\boldsymbol{P}(\mathbf{m m})$ \\
Arica - Parinacota y Tarapacá & 8 & 11,5 & 13,6 & 4807,4 & 19511,4 & $\mathbf{9 , 4}$ & 19,4 & 2,5 \\
Antofagasta & 5 & 54,5 & 12,1 & 6611,4 & 26852,0 & $\mathbf{3 1 , 8}$ & 14,8 & 17,3 \\
Atacama & 11 & 19,8 & 36,9 & 4322,8 & 31684,0 & $\mathbf{1 7 , 1}$ & 15,5 & 26,0 \\
Coquimbo & 8 & 26,7 & 63,9 & 3814,9 & 15638,9 & $\mathbf{5 5 , 2}$ & 14,3 & 160,1 \\
Valparaíso & 6 & 37 & 20,8 & 6794,3 & 28775,7 & $\mathbf{2 1 , 7}$ & 13,8 & 684 \\
Metropolitana & 10 & 50,5 & 23,4 & 6534,8 & 23177,2 & $\mathbf{3 3 , 3}$ & 13,2 & 443,1 \\
\hline O'Higgins & 10 & 17,2 & 10,5 & 3309,1 & 15772,6 & $\mathbf{4 , 9}$ & 12,5 & 557,1 \\
\hline
\end{tabular}

En la tabla d: insectos/humano, $\mathrm{n}$ el número de datos en que se basó la estimación, $i_{t}$ (insectos/hora) es el índice triatomino, $i_{d}$ el porcentaje de infestación domiciliaria $N_{v}$ el número de viviendas, $N$ la población humana, y $T$ y $P$ el promedio anual de temperaturas y precipitaciones totales en cada región.

Tabla 3. Producción promedio de $\mathrm{CO}_{2}$ de Triatoma infestans por temperatura $\left({ }^{\circ} \mathrm{C}\right)$

\begin{tabular}{|lll|}
\hline $\mathbf{T}\left({ }^{\circ} \mathbf{C}\right)$ & $\mathbf{V}_{\mathbf{c o 2}}(\boldsymbol{\mu L} / \mathbf{h})$ & $\mathbf{V}_{\mathbf{c o} 2}(\boldsymbol{\mu L} / \mathbf{h g})$ \\
\hline 10,5 & $67,1 \pm 16,5$ & $257,0 \pm 72,9$ \\
15 & $47,5 \pm 8,3$ & $441,3 \pm 105,3$ \\
23 & $55,2 \pm 14,1$ & $447,2 \pm 85,9$ \\
28 & $76,9 \pm 16,8$ & $617,9 \pm 86,2$ \\
32,5 & $161,4 \pm 39,9$ & $678,6 \pm 155,1$ \\
\hline
\end{tabular}

Las proyecciones del cambio climático sugieren un aumento en $R_{0}$ en el escenario optimista y en el pesimista, moderado de la media $R_{0}$ pero mayor en la mediana $(\mathrm{p}<0,001)$ (Tabla 4$)$. Se obtuvieron valores más altos de $R_{0}$ en la zona central entre Atacama y el norte de la Región de O’Higgins (Figura 2). También aumentó el área con valores $R_{0}$ $\geq 1$ en la zona norte (Figura 3 ). Hubo un aumento de $13,1 \%$ en el área con $R_{0} \geq 1$ con la RCP 2,6 y 
Tabla 4. Estadística descriptiva de $\boldsymbol{R}_{0}$ para el escenario climático actual y escenarios futuros, considerando todos los lugares y donde hay transmisión efectiva $\left(R_{0} \geq 1\right)$

\begin{tabular}{|lcccccc|}
\hline & & Todos & & \multicolumn{3}{c|}{ Transmisión efectiva } \\
& $\boldsymbol{R}_{\mathbf{0}}$ actual & RCP2,6 & $\mathbf{R C P 8 , 5}$ & $\boldsymbol{R}_{\mathbf{0}}$ actual & RCP2,6 & RCP8,5 \\
\hline Promedio \pm 1SD & $2,33 \pm 2,57$ & $2,71 \pm 2,71$ & $2,70 \pm 2,69$ & $4,29 \pm 1,94$ & $4,49 \pm 2,05$ & $4,28 \pm 2,18$ \\
\hline Mediana & 1,010 & 2,603 & 2,407 & 4,706 & 4,616 & 4,299 \\
Moda & 0 & 0 & 0 & 4,715 & 3,826 & 2,385 \\
\hline IQR & {$[0 ; 4,8]$} & {$[0 ; 4,9]$} & {$[0 ; 4,8]$} & {$[3,2 ; 5,1]$} & {$[3,4 ; 5,3]$} & {$[2,7 ; 5,4]$} \\
\hline
\end{tabular}

$\mathrm{SD}=$ desviación estándar; IQR = rango intercuartílico.

el 18,3\% con la RCP 8,5 (Tabla 5). El aumento mayor fué en el extremo norte de Chile, cercano a San Pedro de Atacama. Sin embargo, hubo una expansión en toda el área de estudio. Se identificaron cinco regiones con un valor mayor que el promedio nacional, Tarapacá $\left(R_{0}=2,41\right)$, Antofagasta $\left(R_{0}\right.$ $=2,39)$, Coquimbo $\left(R_{0}=2,41\right)$, Valparaíso $\left(R_{0}=\right.$ $4,34)$ y RM $\left.\left(R_{0}\right)=3,44\right)$. Los mayores cambios en los valores se registraron en Coquimbo y Valparaíso, de 54\% y 26\%, respectivamente (Figura 4).

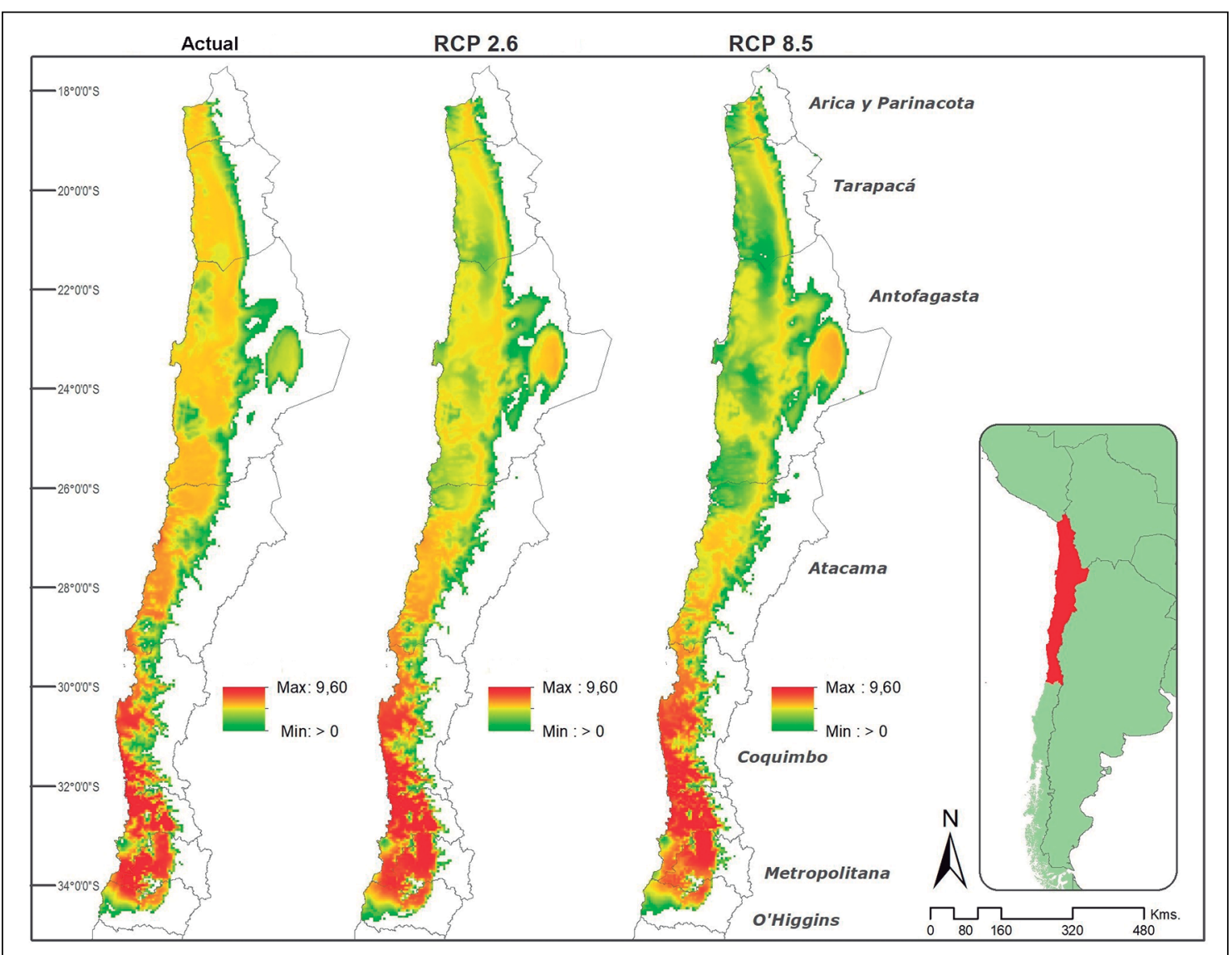

Figura 2. Mapas del número reproductivo estimado de la transmisión de Trypanosoma cruzi en Chile en la condición climática actual y bajo dos escenarios futuros (RCP 2,6 y RCP 8,5). 


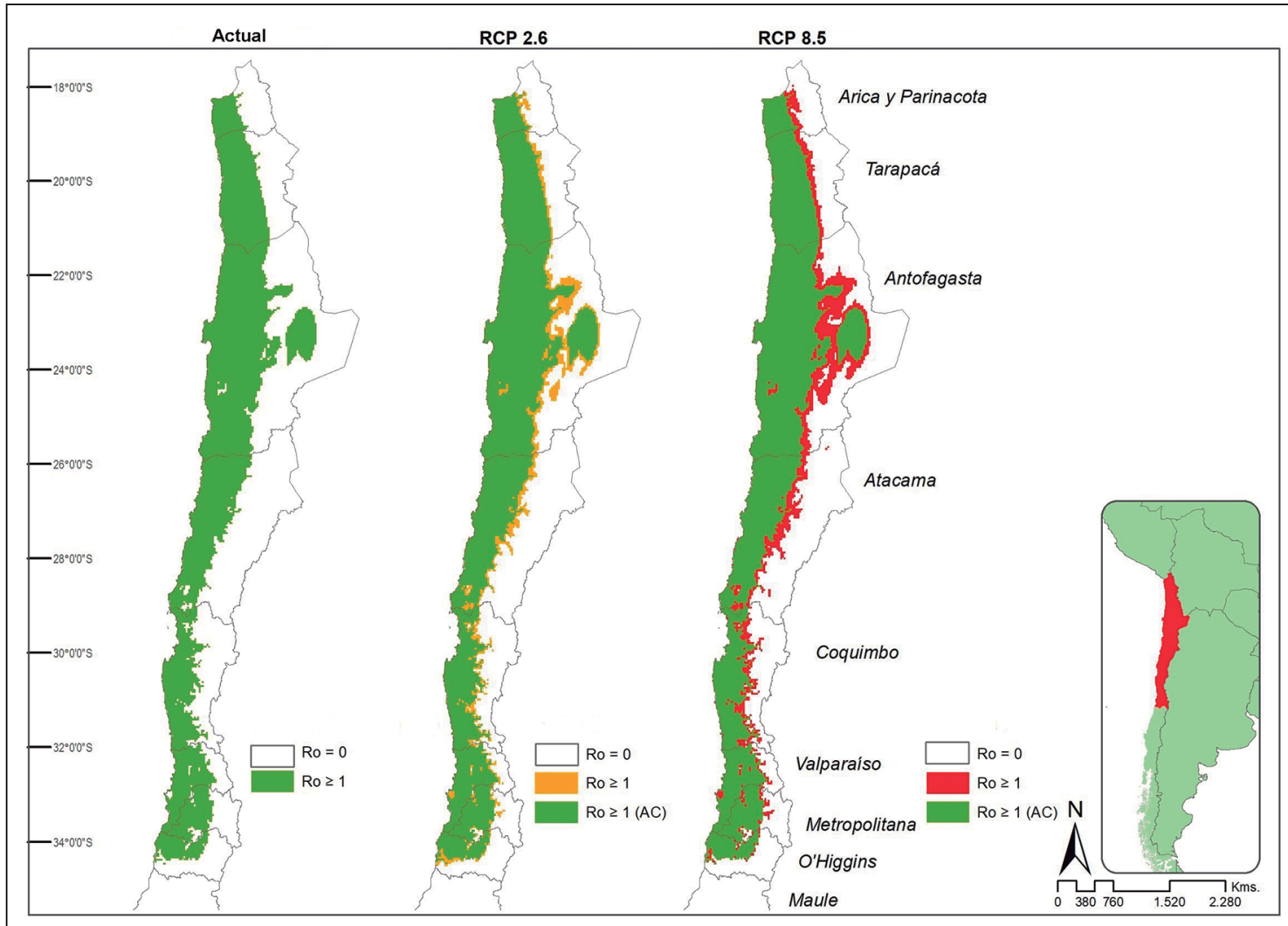

Figura 3. Mapas de las áreas estimadas con transmisión efectiva de Trypanosoma cruzi en Chile en la condición climática actual (verde) y bajo dos escenarios futuros RCP 2,6 (amarillo) y RCP 8,5 (rojo).

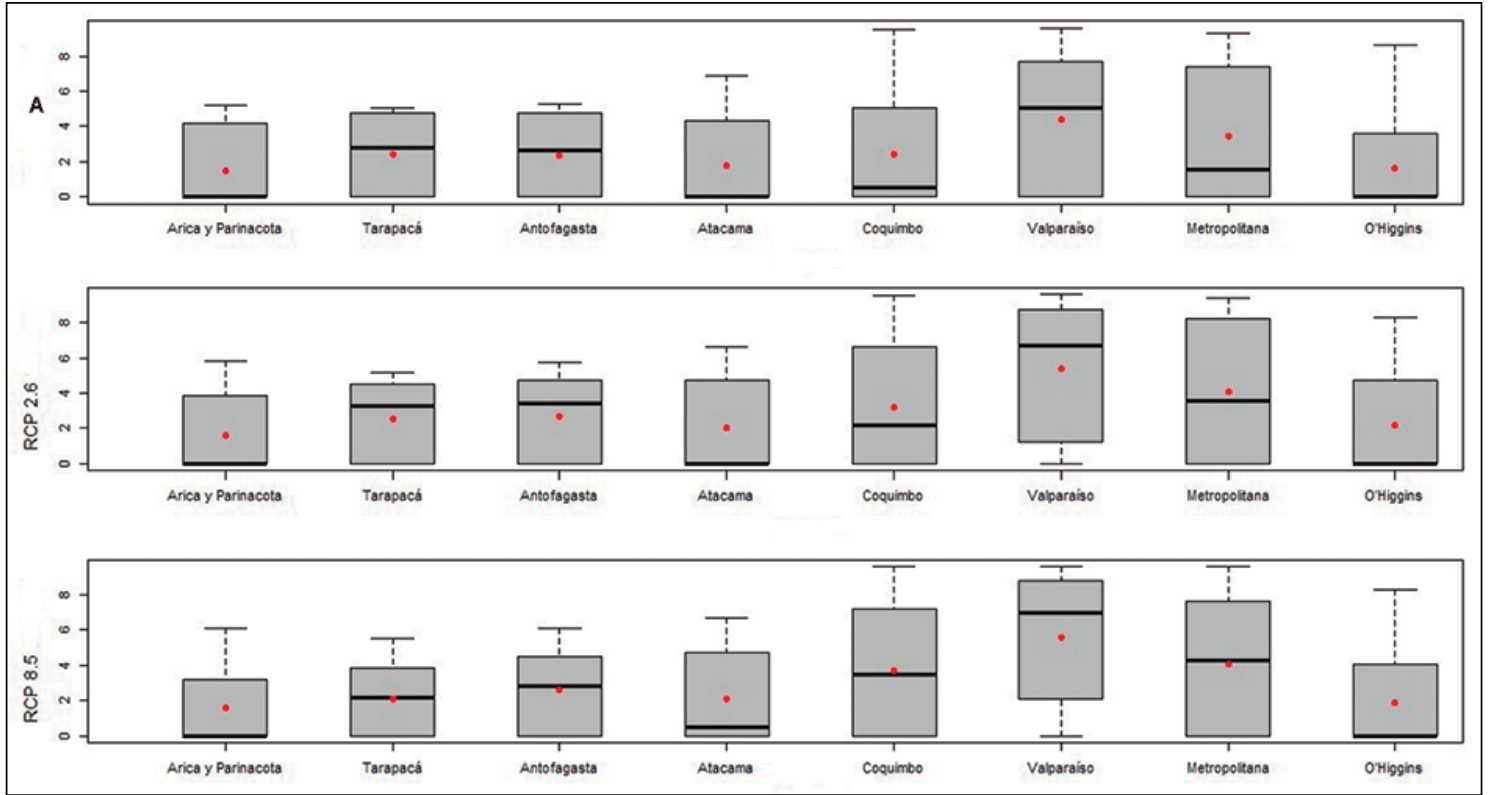

Figura 4. Números reproductivos estimados para las regiones del área endémica de la enfermedad de Chagas en Chile. 


\section{Tabla 5. Área $\left(\mathrm{km}^{2}\right)$ con $\left(R_{0} \geq 1\right)$ y $\sin \left(R_{0}<1\right)$ transmisión de Chagas en la situación actual y escenarios futuros}

\begin{tabular}{|lccc|}
\hline & Actual & RCP 2,6 & RCP 8,5 \\
\hline$R_{0} \geq 1$ & 174880,3 & 197727,9 & 206946,6 \\
$R_{0}<1$ & 204559,4 & 181714,0 & 172496,4 \\
\hline
\end{tabular}

\section{Discusión}

En este estudio estimamos el riesgo de transmisión vectorial de la enfermedad de Chagas basados en parámetros de T. infestans, datos poblacionales humanos y datos climáticos de Chile. A pesar que en Chile se ha certificado el corte de transmisión vectorial por esta especie, aún se recibe en el Instituto de Salud Pública un número elevado de especímenes, con un porcentaje de infección por T. cruzi cercano a $40 \%$, cada año se reporta mayor número de intromisiones domiciliarias y un mayor número de focos silvestres ${ }^{22}$, hechos que junto a la existencia de un gran reservorio animal y vectores silvestres de distribución similar, sustentan la mantención del riesgo de transmisión vectorial.

La distribución del número reproductivo fue muy asimétrica como consecuencia de muchas zonas con $R_{0}=0$. En estudios previos en Chile, se propuso $R_{0}=2,86$ en $1992(38)$ y $R_{0}=1,52$ en $2017^{17}$. En otros países, se estimó un valor $R_{0}=1,25$ en Brasil $^{39}$ y valores más altos en Colombia ${ }^{16}$, donde hay otros vectores y donde la situación es muy diferente y compleja. Los valores cercanos al valor mediano son consistentes con el estado endémico de la enfermedad de Chagas en Chile. La respuesta funcional de la tasa de mortalidad de $T$. infestans fue consistente con el comportamiento de la mortalidad de vectores, con alta mortalidad a altas y bajas temperaturas y menor mortalidad en un área que coincide con la temperatura preferida por esta especie ${ }^{34}$. Además, los valores son consistentes con otros valores publicados para esta especie ${ }^{12}$. La densidad estimada, coincide con aquellos lugares con mayor presencia de vectores $^{8,11}$. La relación directa entre la tasa de picada y el gasto de energía, es una suposición razonable ya utilizada en insectos vectores y otros grupos de artrópodos ${ }^{33}$ y fue consistente con los valores publicados para esta especie $\mathrm{e}^{15,40}$.

El $R_{0}$ más alto corresponde al área central,
Valparaíso y la Región Metropolitana. Las regiones extremas del área endémica tuvieron valores más bajos, Arica y Parinacota y la Región de O’Higgins. Esto concuerda con la distribución de casos de la enfermedad de Chagas ${ }^{8,24}$ y de focos remanentes e intrusión de hogares por T. infestans reportados por el MINSAL. Sin embargo, el MINSAL reporta también altas incidencias de enfermedad de Chagas también en la Región de Antofagasta ${ }^{22}$.

Podría haber un aumento significativo de riesgo de la enfermedad de Chagas en el escenario optimista RCP 2,6 y en el escenario RCP 8,5, en comparación con el escenario actual, lo que se hace más evidente al comparar las medianas que aumentan a más del doble. Los valores más altos de $R_{0}$ se concentraron en el sector costero y la depresión intermedia, coincidiendo con los climas desértico, semiárido y septentrional de esta región. El aumento se explicaría por los cambios en la temperatura esperados para Chile. Se espera un aumento desde $0,5^{\circ} \mathrm{C}$ en la costa hasta $5^{\circ} \mathrm{C}$ en la Cordillera de los Andes, en el centro y norte del país ${ }^{41}$. Esta área también concuerda con el área donde hay vectores y casos de la enfermedad de Chagas $^{8}$.

El área con riesgo de transmisión efectiva para la enfermedad de Chagas mostró un aumento de $18,3 \%$ para RCP 8,5 y $13,1 \%$ para RCP 2,6 . Otros estudios han propuesto que se produciría una disminución muy pequeña en el hábitat adecuado para $T$. infestans y también para el vector silvestre Mepraia spinolai en ambos escenarios ${ }^{8,24}$. Estos estudios se han realizado con datos de presencia, lo que difiere de la aproximación de nuestro estudio, lo que explicaría la diferencia entre estos resultados. Los aumentos en $R_{0}$ pueden ocurrir por aumentos en la densidad, aumentos en la tasa de picada o disminuciones en la mortalidad. Aunque, se debe considerar que este tipo de modelos no captura la complejidad de los microambientes como los refugios ${ }^{42,43}$. Por ejemplo, los microambientes que encuentran las vinchucas en ambientes estables como viviendas humanas y gallineros les proporcionan posibilidad de reproducción y alimentación que favorece su elección. Así por ejemplo se han reportado en T. infestans mayores volúmenes de ingesta de sangre y mayor fecundidad en insectos asociados a gallineros y a la vivienda humana revelando el compromiso entre los componentes de la adecuación biológica y la elección de micro-sitios ${ }^{44}$. 
El aumento en el área con riesgo efectivo $\left(R_{0} \geq 1\right)$ y en $R_{0}$ es relevante, considerando la identificación de colonias silvestres de T. infestans desde $2003^{45} \mathrm{y}$ el aumento en el número de intromisiones de esta especie en hábitats domésticos ${ }^{17}$. Suponiendo un corte en la cadena de transmisión del vector, la enfermedad de Chagas podría continuar presentando casos hasta por 60 años ${ }^{22}$, lo que reitera el cuidado que debe existir en el mantenimiento del control vectorial domiciliario y en focos silvestres.

El valor obtenido para $R_{0}$ es la primera estimación empírica de este tipo en Chile, incorporando la distribución espacial e identificando las áreas de mayor riesgo de transmisión sobre las cuales se debe enfocar la vigilancia y el control del vector. Si no se mantiene el control de T. infestans y los brotes de vectores silvestres, la enfermedad de Chagas continuará presentando casos independientemente del escenario de cambio climático futuro.

\section{Referencias}

1. Dobson A. Climate variability, global change, immunity, and the dynamics of infectious diseases. Ecology 2009; 90 (4): 920-7.

2. Gage KL, Burkot TR, Eisen RJ, Hayes EB.Climate and vector borne diseases. Am J Prev Med 2008; 35 (5): 43650.

3. Gubler DJ.Vector-borne diseases. Rev Sci Tech 2009; 28: 583-8.

4. Hartemink NA, Purse BV, Meiswinkel R, Brown HE, de Koeijer A, Elbers ARW, et al. Mapping the basic reproduction number (R0) for vector-borne diseases: A case study on bluetongue virus. Epidemics 2009; 1 (3): 153-61.

5. Parham PE, Michael E. Modeling the Effects of Weather and Climate Change on Malaria Transmission. Env Health Pers 2010; 118 (5): 620-6.

6. Figueroa D, Scott S, Hamilton-West C, González CR, \& Canals M. Mosquitoes: Diseases vectors in context of climate change. Parasitol Latinoam 2015; 64 (2): 42-53.

7. Canals M, Cattan PE. Zoonosis emergentes transmitidas por vectores artrópodos en un mundo marcado por el cambio global. Parasitol Latinoam 2016; 65 (1): 42-53.

8. Tapia-Garay V, Figueroa DP, Maldonado A, Frías-Laserre D, González CR, Parra A, et al. Assessing the risk zones of Chagas' disease in Chile in a world marked by global climatic change. Mem Inst Oswaldo Cruz 2018; 1-6. doi: 10.1590/0074-02760170172.

9. Kovats RS, Campbell-Lendrum DH, McMichael AJ, Woodward A, Cox JStH. Early effect of climate change: do they include changes in vector-borne diseases? Phil Trans R Soc Lond B 2001; 356: 1057-68.

10. Canals M, Cattan P, Ehrenfeld M, Torres P. Poblaciones experimentales de T. infestans: efectos de condiciones ambientales variables. Parasitol Día 1992; 16: 72-7.

11. Canals M, Cattan E, Ehrenfeld M. Algunas estimaciones numéricas de la importancia epidemiológica de los vectores de la enfermedad de Chagas en Chile. Parasitol Día 1993; 17 (3/4): 79-86.

12. Canals M, Ehreneld M, Solís R, Cruzat L, Pinochet A, Tapia C, et al. Biología comparada de Mepraia spinolai en condiciones de laboratorio y Terreno: cinco años de estudio. Parasitol Día 1998; 22 (3-4): 72-8.

13. Canals LM, Ehrenfeld HM, Cattan APE. Situación de Mepraia spinolai, vector silvestre de la enfermedad de Chagas en Chile, en relación con otros vectores desde la perspectiva de sus fuentes de alimentación. Rev Med Chile 2000; 128 (10): 110812.

14. Ehrenfeld M, Canals M, Cattan PE. Population parameters of Triatoma spinolai under different environmental conditions and densities. J Med Entomol 1998; 35 (5): 740-4.

15. de la Vega GJ, Medone P, Ceccarelli S, Rabinovich J, Schilman PE. Geographical distribution, climatic variability and thermo-tolerance of Chagas disease vectors. Ecography 2015; 38 (8): 851-60.

16. Cordovez JM, Rendon LM, González C, Guhl F. Using the basic reproduction number to assess the effects of climate change in the risk of Chagas disease transmission in Colombia. Acta Trop 2014; 129: 74-82.

17. Canals M, Cáceres D, Alvarado S, Canals A, Cattan PE. Modeling Chagas disease in Chile: From vector to congenital transmission. Biosystems 2017; 156-157: 63-71.

18. Ostfield RS, Glass GE, Keensing F. Spatial epidemiology: an emerging (or re-emerging) discipline TREE 2005; 20 (6): 328-36.

19. Hotez PJ, Bottazzi ME, Franco-Paredes C, Ault SK, Periago MR. The Neglected Tropical Diseases of Latin America and the Caribbean: A Review of Disease Burden and Distribution and a Roadmap for Control and Elimination. PLoS Negl Trop Dis Sep 2008; 24; 2 (9): e300.

20. Hotez PJ, Dumonteil E, Woc-Colburn L, Serpa JA, Bezek S, Edwards MS, et al. Chagas disease: The New HIV/AIDS of the Americas. PLoS Negl Trop Dis 2012; 6 (5): e1498. doi:10.1371/journal.pntd.0001498. 
21. Cucunubá ZM, Okuwoga O, Basáñez MG, Nouvellet P. Increased mortality attributed to Chagas disease: a systematic review and meta-analysis. Parasit Vectors 2016; 9: 42. DOI 10.1186/s13071-016-1315-x.

22. Canals M, González C, Canals L, Canals A, Cáceres D, Alvarado S, et al. ¿Qué dicen los números de la evolución temporal de la enfermedad de Chagas? Rev Chilena Infectol 2017; 34 (2): 120-7.

23. Hernandez J, Núñez I, Bacigalupo A, Cattan PE. Modeling the spatial distribution of Chagas disease vectors using environmental variables and people's knowledge. Int J Health Geogr 2013; 12: 29. https://doi. org/10.1186/1476-072X-12-29.

24. Garrido R. Impacto del cambio climático en la distribución geográfica de dos vectores silvestres de la enfermedad de Chagas en Chile, Mepraia spinolai y Mepraia gajardoi (Hemiptera: Reduviidae) [Magíster en Ciencias Biológicas]. [Chile]: Universidad de Chile. 2017.

25. Martínez R, Ahumada C, Contreras MC, Villarroel F, Rojas A, Schenone H. Chagas disease in Chile. Rural sections. Domiciliary triatomid infestation and Trypanosoma cruzi infection of the vector and of domestic mammals from the I Region (1982-1983). Bol Chil Parasitol 1983; 38: 70-2.

26. Burchard L, Cornejo L, Cruz L, Contreras MC, Villarroel F, Rojas A, et al. Chagas disease in Chile. Domiciliary triatomid infestation and Trypanosoma cruzi infection of the vector and of domestic mammals from the II Region. Bol Chil Parasitol 1983; 39 (1-2): 17-9.

27. Bertoglia J, Rodríguez J, Gordillo N, Mendoza J, Contreras MC, Rojas J, et al. Chagas disease in Chile. Rural sections. Trypanosoma cruzi infection of domestic mammals and new contributions to the knowledge of domiciliary infestation in the III Region. Bol Chil Parasitol 39 (1-2): 20-3.

28. Correa V, Zúñiga J, Briceño J, Contreras MC, Aranda JC,Valdés J, et al. Chagas disease in Chile. Rural sections. Domiciliary infestation by Triatominae, rates of Trypanosoma cruzi infection in these and new contributions to the knowledge on Chagasic infection of domestic mammals from the Region IV(1982-1983). Bol Chil Parasitol 39 (1-2): 24-7.

29. Flores B, Hernández G, Lepe A, Contreras MC, Sandoval L, Villarroel F, et al. Epidemiología de la enfermedad de Chagas en Chile. Sectores Rurales. Infestación triatomídea domiciliaria e infección por Trypanosoma cruzi del vector y de mamíferos domésticos de la V Región, 1983. Bol Chil Parasitol 39(3-4): 62-4.

30. Villarroel F, Rojas A, Contreras MC, Schenone H. Epidemiología de la enfermedad de Chagas en Chile. Sectores Rurales. Infestación triatomídea domiciliaria e infección por Trypanosoma cruzi de los vectores y de mamíferos domésticos de la Región Metropolitana, 1982-1984. Bol Chil Parasitol 39(3-4): 65-8.

31. Venegas L, Rojas A, Villarroel F, Contreras MC, Sandoval L, Schenone H. Epidemiología de la enfermedad de Chagas en Chile. Sectores Rurales. Infestación triatomídea domiciliaria e infección por Trypanosoma cruzi del vector y de mamíferos domésticos de la VI Región del Libertador General Bernardo O’Higgins, 1983. Bol Chil Parasitol 39 (3-4): 69-71.

32. Rolandi C, Schilman PE. Linking global warming, metabolic rate of hematophagous vectors, and the transmission of infectious diseases 2012; 3: 75. doi. 10.3389/ fphys.2012.00075.

33.- Canals L, Figueroa DP, Torres-Contreras H, Veloso C, Canals M. Are mealworm (Tenebrio molitor) diets in concordance with energetic requirements of small mygalomorph spiders (Paraphysa sp.)? J Exot Pet Med 2012; 21: 203-6.

34. Canals M, Solís R, Valderas J, Ehrenfeld M, Cattan PE. Preliminary studies on Temperature selection and activity cycle of Chilean vectors of the Chagas disease. J Med Entomol 1997; 34 (1): 11-7.

35. Canals M, Veloso C, Moreno L, Solís R. Low metabolic rates in primitive hunters and weaver spiders. Physiol Entomol 2015; 40: 232-8.

36. Gillooly JF. Effects of Size and Temperature on Metabolic Rate. Science 2001; 293 (5538): 2248-51.

37. Hijmans RJ, Cameron SE, Parra JL, Jones PG, Jarvis A. Very high resolution interpolated climate surfaces for global land areas. Int J Climatol 2005; 25 (15): 1965 78.

38. Canals M, Cattan PE. Dinamica de transmisión de la infección chagásica en Chile: Modelo y simulación. Rev Med Chile 1992; 120 (12): 1359-65.

39. Massad E. The elimination of Chagas' disease from Brazil. Epidemiol Infect 2008; 136 (9): 1153-64.

40. Rolandi C, Iglesias MS, Schilman PE. Metabolism and water loss rate of the haematophagous insect Rhodnius prolixus: effect of starvation and temperature. J Exp Biol 2014; 217 (24): 4414-22.

41. Garreaud R. Cambio Climático: Bases Físicas e Impactos en Chile. Rev Tierra Adentro INIA. 2011; 93.

42. Schofield CJ, Jannin J, Salvatella R. The future of Chagas disease control. Trends Parasitol 2006; 22 (12): 583-8.

43. Telleria J, Tibayrenc M. American trypanosomiasis: Chagas disease one hundred years of research. 2017. 2da ed. Boston, MA: Elsevier.

44. Gürtler RE, Fernández MP, Cecere MC, Cohen JE. Body size and hosts of $T$. infestans populations affect the 
bloodmeal contents and fecundity in rural northwestern Argentina. PLoS Negl Trop Dis 201711 (12): e0006097. https://doi.org/10.1371/journal.pntd.0006097.

45. Bacigalupo A, Segura JA, García A, Hidalgo CJ, Galuppo
GS, Cattan PE. Primer hallazgo de vectores de la enfermedad de Chagas asociados a matorrales silvestres en la Región Metropolitana, Chile. Rev Med Chile 2006; 134 (10): 1230-6. 